16. Chia YT, Chua S, Thai AC, et al. Gestational diabetes. Obstetric and neonatal outcome in 411 cases. Singapore Med J. 1996;37:591-594.

17. Seshiah V, Balaji V, Balaji Madhuri S, Panneerselvam A, Kapur A. Pregnancy and Diabetes Scenario around the World: India. Int J GynaecolObstet. 2009;104(suppl 1): S35-S38.

18. Zargar AH, Sheikh MI, Bashir MI, et al. Prevalence of gestational diabetes mellitus in Kashmiri women from the
Indian subcontinent. Diabetes Res Clin Pract. 2004;66(2):139-145. Pg - 18.

19. Divakar H, Tyagi S, Hosmani P, Manyonda IT. Diagnostic criteria influence prevalence rates for gestational diabetes: implications for interventions in an Indian pregnant population. Perinatology. 2008;10(6):155-161.

20. Franks PW, Looker HC, Kobes S, et al. Gestational Glucose Tolerance and Risk of Type 2 Diabetes in Young Pima Indian Offspring. Diabetes. 2006;55(2):460-465.

\title{
Observations on community associated methicillin resistant Staphylococcus aureus carriage
}

\author{
Bhawana Jain ${ }^{a}$, Jyotsna Agarwal ${ }^{b, *}$, Mastan Singh ${ }^{c}$ \\ ${ }^{a}$ Research Scientist II (Medical), Department of Microbiology, King George’s Medical University, Lucknow, \\ Uttar Pradesh 226003, India \\ ${ }^{\mathrm{b}}$ Professor, Department of Microbiology, King George's Medical University, Lucknow, Uttar Pradesh 226003, India \\ ' Professor, Head of Department, Department of Microbiology, King George's Medical University, Lucknow, \\ Uttar Pradesh 226003, India
}

\section{A R T I C L E I N F O}

Article history:

Received 18 February 2013

Accepted 21 April 2013

Available online 30 April 2013

Keywords:

Antibiotic susceptibility

Community associated MRSA

Staphylococcal carriage sites

\begin{abstract}
A B S T R A C T
Background \& Objectives: Community associated methicillin resistant Staphylococcus aureus (CA-MRSA) infections are becoming increasingly important. Present study was conducted to know CA-MRSA prevalence in apparently healthy individuals, colonization sites and antibiotic susceptibility pattern.

Methods: It was a prospective, hospital based study in which 200 healthy individuals (accompanying the patients attending outdoor services at a tertiary care center) with no history of recent hospitalization/surgery, and antibiotic intake were randomly enrolled as study subjects. A total of 600 samples one each from nose, throat and axilla, were collected. 100 admitted patients were enrolled as controls to look for acquisition of hospital acquired MRSA (HA-MRSA).

Results: A total of 204 S. aureus isolates were recovered from 116 subjects in the study group; maximum yield was from throat, followed by anterior nares. Of these, $41.2 \%(84 / 204)$ were MRSA as detected by oxacillin MIC by agar dilution method. Over all CA-MRSA colonization at one or more body sites was found in $23.5 \%(47 / 200)$ of study subjects. The antibiotic susceptibility testing showed $25 \%$ of CAMRSA to be resistant to clindamycin.

Interpretation and conclusions: A considerably high proportion of the population $(23.5 \%)$ in study group was colonized with CA-MRSA and throat was the commonest site for both S. aureus and MRSA carriage.

Copyright @ 2013, INDIACLEN. Publishing Services by Reed Elsevier India Pvt Ltd. All rights
\end{abstract} reserved.

\footnotetext{
* Corresponding author. Tel.: +91522 2668484.

E-mail addresses: jyotsna.kgmu@gmail.com (J. Agarwal)

2213-3984/Copyright @ 2013, INDIACLEN. Publishing Services by Reed Elsevier India Pvt Ltd. All rights reserved. 


\section{Introduction}

Staphylococcus aureus (SA) is one of the most common bacterial infections encountered in day-to-day practice by clinicians. In spite of best of the efforts to treat and control it, newer drug resistant SA variants are posing a challenge. Over the past 50 years, Methicillin Resistant S. aureus (MRSA) has been a major threat to human health either as hospital acquired (HA-MRSA) or recently as community associated (CA-MRSA) pathogen, ${ }^{1,2}$ owing to its ability to colonize at various body sites and being multi-drug resistant as well. CA-MRSA refers to MRSA infection with onset in the community in an individual lacking established MRSA risk factors, such as recent hospitalization, surgery, residence in a long-term care facility, receipt of dialysis or presence of invasive medical devices. ${ }^{3}$ CA-MRSA has emerged very rapidly since it first appeared in late 1990s, affecting virtually every geographical region, be it rural or urban, causing not only relatively minor skin and soft tissue infection but also quite severe pneumonitis, necrotizing fasciitis and osteomyelitis. ${ }^{4}$ Clinical, epidemiological, molecular and antibiotic resistance profiles show that CA-MRSA strains are different from those of hospital acquired MRSA (HA-MRSA). ${ }^{5}$ CA-MRSA remains susceptible to several non-beta lactam groups of antimicrobial agents unlike HA-MRSA. ${ }^{6}$ Moreover epidemiologically, CA-MRSA resembles more with methicillin sensitive SA (MSSA) rather than HA-MRSA, with crowding, repeated physical contact, poor hygiene and skin trauma being the main conditions leading to colonization and infection. ${ }^{4}$ Relatively little is known regarding colonization pattern of CA-MRSA while nasal carriage of HA-MRSA is strongly related to its infection. ${ }^{7}$ Worldwide studies are going on to reveal colonization sites and their association with infection. Literature search did not give much data on colonization pattern of CAMRSA and antibiotics susceptibility pattern from North India too. Therefore this study was planned with the aim of observing the prevalence of colonization by CA-MRSA at various body sites and their antimicrobial susceptibility pattern in this part of the world. Locally generated data can be used for policy decision regarding CA-MRSA screening and guidelines for empirical treatment and decolonization.

\section{Methods}

It was a prospective study, carried out between October 2006 and November 2007 in a tertiary hospital associated with King George's Medical University, Lucknow. The Institute's Ethics Committee approved the study. A total of 200 apparently healthy individuals, accompanying the patients attending outdoors for the first time, were randomly enrolled after obtaining written informed consent. Inclusion criteria included no history of hospitalization/surgery/antibiotic intake within last one-year. Three swabs were taken from each individual - nasal, axillary and throat as per method described in standard texts. To compare this study group with hospital acquired MRSA (HA-MRSA); 100 randomly selected indoor patients from a surgical ward were enrolled as controls. One nasal swab each was collected from these patients at the time of admission to look for MRSA carriage and those found positive were excluded from further study. From remaining patients another nasal swab was taken $72 \mathrm{~h}$ after admission to look for acquisition of HA-MRSA (Fig. 1).

All swabs were immediately brought to the laboratory and inoculated on mannitol salt agar. Plates were incubated at $37^{\circ} \mathrm{C}$ for overnight in ambient air incubator. Colonies were identified by colony morphology, Gram staining and biochemicals if needed. Staphylococci thus isolated were subjected to slide and tube coagulase tests to pick outS. aureus. ${ }^{8}$ Methicillin resistance was determined by oxacillin MIC using agar dilution method. Reference grade oxacillin powder was obtained from Hi Media Laboratories, India. Mueller Hinton agar (Microexpress, Tulip) with $2 \% \mathrm{NaCl}$ was used; plates were prepared containing doubling dilutions of oxacillin ranging from $0.125 \mathrm{mg} / \mathrm{l}$ to $64 \mathrm{mg} / \mathrm{l}$. The final inoculum used was $10^{4}-10^{5} \mathrm{cfu} / \mathrm{spot}$. They were incubated at $30^{\circ} \mathrm{C}$ for full $24 \mathrm{~h}$. S. aureus strain ATCC 29213 was used as control with each batch of tests. MIC for control strain was within one, two-fold dilution step of $0.25 \mathrm{mg} / \mathrm{l}$ of oxacillin. MIC was read as the point of complete inhibition of growth. Trailing end points or reduced numbers of colonies other than a single colony in the spot were not taken as complete inhibition. Oxacillin MIC of $\leq 2 \mathrm{mg} / \mathrm{l}$ was taken as susceptible, that of $\geq 4 \mathrm{mg} / \mathrm{l}$ as resistant. ${ }^{9} \mathrm{~S}$. aureus showing oxacillin resistance were subjected to mecA gene detection by conventional PCR method. DNA preparation was done from a single colony of oxacillin resistant strain of $\mathrm{S}$. aureus after overnight growth at $37{ }^{\circ} \mathrm{C}$ in Brain Heart Infusion Broth. ${ }^{10} \mathrm{PCR}$ reaction was set to detect mecA gene from the prepared DNA using Forward primer 5'-AAAATCGATGGTAAAGGTTGGC-3' and Reverse primer $5^{\prime}$-AGTTCTGCAGTACCGGATTTTGC- $3^{\prime}{ }^{11}$ Reaction conditions and master mix was as per Siripornmongcolchai $\mathrm{T}$ et $\mathrm{al}^{10}$

Antibiotic susceptibility pattern of MRSA strains thus obtained was checked using Kirby Bauer method; antibiotic disks used were vancomycin $5 \mu \mathrm{g}$ (Hi Media Laboratories, Mumbai, India); ciprofloxacin $5 \mu \mathrm{g}$; clindamycin $2 \mu \mathrm{g}$; erythromycin $15 \mu \mathrm{g}$ and gentamicin $10 \mu \mathrm{g}$ (Span Diagnostics Ltd., Surat, India). Before use each batch of disks was tested with standard strains of S. aureus ATCC 29213. Zone diameters were interpreted as per CLSI recommendations. ${ }^{9}$

\subsection{Statistics}

Stata 9.2 statistical software was used for calculations. The proportions are expressed in terms of $\%$ and $95 \%$ CI of the proportions are provided. Chi square statistics were used to compare the significance between the proportions and Fisher's exact $p$ values are reported.

\section{Results}

Amongst cases, $58.0 \%$ (116/200) of individuals were colonized by S. aureus; of which $57 \%$ (67/116) were colonized at more than one body site; colonization at one or more body sites by CA-MRSA was $23.5 \%$ (47/200). Throat was the single commonest site for S. aureus (107/200; 53.5\%) as well as MRSA carriage (44/200; 22\%) followed by anterior nares (71/200; $35.5 \%$ S. aureus and $27 / 200$; 13.5\% MRSA) (Fig. 1). Of 116 subjects colonized with S. aureus, throat swab picked up 107 (proportion 92.2\%, 95\%CI 86-96) as 


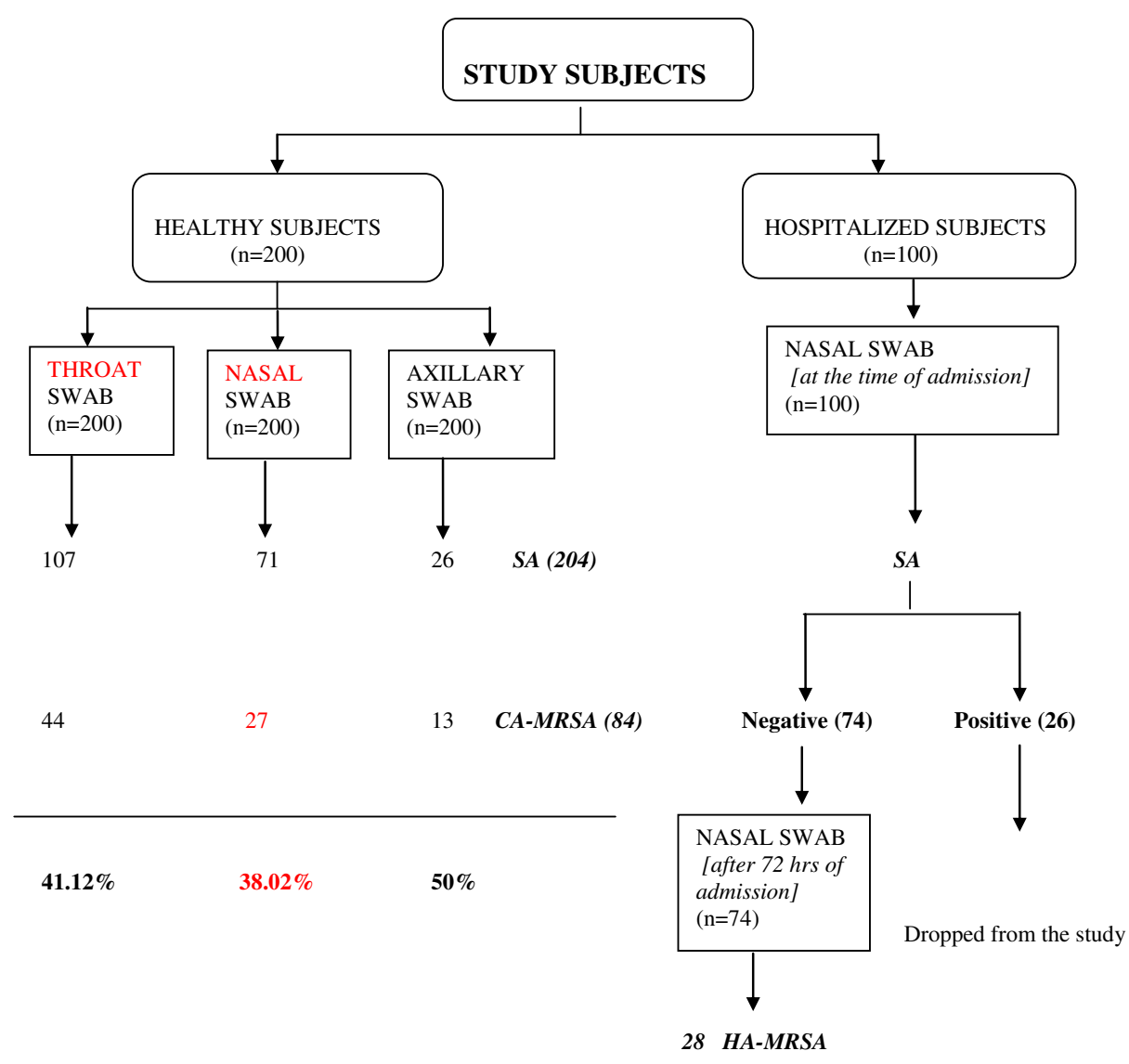

Fig. 1 - Study design and final outcome. SA = Staphylococcus aureus, CA-MRSA = Community associated methicillin resistant Staphylococcus aureus, HA-MRSA = Hospital acquired methicillin resistant Staphylococcus aureus.

against 71 and 26 from anterior nares and axilla (proportion $61.2 \%, 95 \%$ CI $52-70 ; 22.4 \%, 95 \%$ CI $15-31)$. None of the individuals were carrying $S$. aureus in axilla alone (Table 1). Axilla had maximum percentage $(13 / 26,50 \%)$ of CA-MRSA among SA isolates and Nose harbored the least percentage (27/71, 38.02\%) (Fig. 1). Amongst control group, 26 of the 100 patients enrolled carried MRSA in their nose at the time of admission hence they were excluded from the study. Of remaining, 37.8\% (28/74) patients yielded MRSA when swabbed $72 \mathrm{~h}$ after admission. All of the $S$. aureus strains showing oxacillin resistance by phenotypic method were also having mecA gene detected by PCR. The antibiotic sensitivity profile of these HA-MRSA revealed $100 \%$

Table 1 - Colonization by S. aureus \& CA-MRSA at various body sites amongst cases $(n=116)$.

S. aureus CA-MRSA

Single site colonization only

Throat only

Nose only

Axilla only

\begin{tabular}{rr}
41 & 19 \\
8 & 3 \\
0 & 0 \\
41 & \\
4 & 12 \\
1 & 1 \\
21 & 0 \\
116 & 12 \\
\hline
\end{tabular}

Colonization at multiple body sites

Throat \& Nose

Throat \& Axilla

Nose \& Axilla

Throat, Nose \& Axilla

Total resistance to ciprofloxacin and $85.6 \%$ resistant to clindamycin as compared to $22.6 \%$ and $25 \%$ resistant to ciprofloxacin and clindamycin in CA-MRSA isolates respectively, this difference was statistically significant at $p<0.0001$ for ciprofloxacin, clindamycin and gentamicin and at $p=0.0007$ for erythromycin (Fig. 2).

\section{Discussion}

Prevention of CA-MRSA infections and controlling their spread in community and spread to hospital is a critical

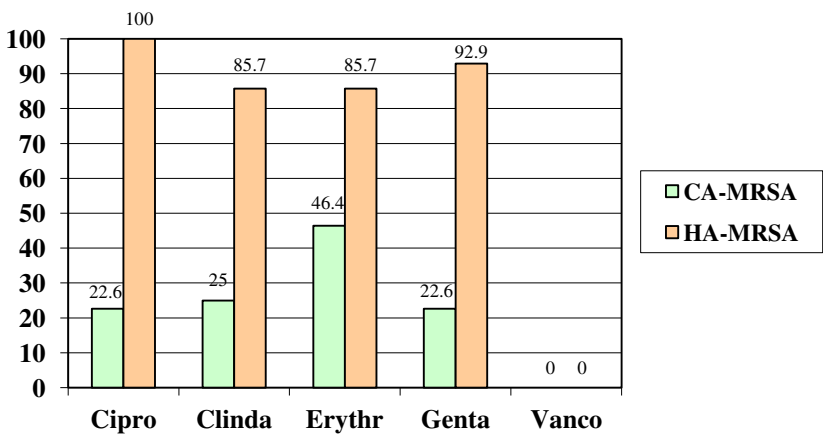

Fig. 2 - Resistance to antibiotics CA-MRSA vs. HA-MRSA. All values are in \%; Cipro = Ciprofloxacin; Clinda = Clindamycin; Erythr = Erythromycin; Genta = Gentamicin; Vanco = Van comycin. 
issue. Significant observation in this study was the large number $(116 / 200,58 \%)$ of apparently healthy individuals from community harboring S. aureus strains while $47 / 200$ i.e. $23.5 \%$ were colonized with MRSA, presumably CA-MRSA as per the study inclusion criteria. A previous study estimates prevalence of CA-MRSA to be around $18.1 \%$ of total SA positives in east Delhi area ${ }^{12}$ and $3.89 \%$ in children of 5-15 years age group in a study from Chandigarh. ${ }^{13}$ We are reporting higher rate of CA-MRSA carriage in comparison to other studies from India, the reasons of which could be that the population we chose to screen was an adult population attending the hospital. Besides, previous studies have not taken a throat swab and hence some of the S. aureus carriage may have been missed by them. However genotypic study to confirm CAMRSA status is required and this is a limitation of our study. Sites of colonization are important as a potential source of subsequent infection to the host and as a reservoir for organism. Previous studies have shown anterior nares to be the most consistent area from where $S$. aureus can be isolated. ${ }^{14}$ In present study however S. aureus was isolated most frequently from throat. Similar findings have been reported in a study carried out in Sweden where they found $40 \%$ of the patients and $54 \%$ of the staff positive for S. aureus in the throat, compared to $31 \%$ and $36 \%$, respectively, in the anterior nares. ${ }^{15}$ Our results demonstrate that $\mathrm{S}$. aureus was infrequently carried in anterior nares alone; if anterior nares were colonized, with few exceptions, it was present in throat also (Table 1). Throat seems to be a better choice if only one site is to be sampled. This could have important prophylactic implications since decolonization of only nasal carriers has been tried with different patients groups to limit MRSA spread. The source of bacteria in many of these cases could be other carriage sites on the patients.

In the control group acquisition of HA-MRSA was $37.8 \%$; some isolates may have been missed as only nasal swab was taken for this group. On comparing the sensitivity pattern we found HA-MRSA to be significantly more resistant to all groups of antibiotics (Fig. 2). Earlier, sensitivity to clindamycin was taken as a surrogate marker of CA-MRSA detection ${ }^{16}$; in present study however $25 \%$ of CA-MRSA was found resistant to clindamycin. This trend needs to be closely followed up to determine appropriate empirical treatment policy for CAMRSA infections.

\section{Conflicts of interest}

All authors have none to declare.

\section{REFERENCES}

1. Chambers HF. The changing epidemiology of Staphylococcus aureus. Emerging Infect Dis. 2001;7:178-182.

2. Shopsin B, Kreiswirth B. Molecular epidemiology of methicillin resistant Staphylococcus aureus. Emerging Infect Dis. 2001; 7:322-326.

3. Chih- YC, Wong WW, Fung CP, Yu KW, Liu C. Epidemiology of community acquired Staphylococcus aureus bacteremia. J Microbiol Immunol Infect. 2004;37:16-23.

4. Wallin TR, Hern HG, Frazee BW. Community-associated methicillin resistant Staphylococcus aureus. Emerg Med Clin N Am. 2008;26:431-455.

5. Naimi TS, LeDell KH, Como-Sabetti K, et al. Comparison of community- and health care-associated methicillin resistant Staphylococcus aureus infection. JAMA. 2003;290:2976-2984.

6. Kuehnert MJ, Kruszon-Moran D, Hill HA, McQuillan G, McAllister SK, Fosheim G. Prevalence of Staphylococcus aureus colonization in the US 2001-2002. J Infect Dis. 2006;193:172-179.

7. Graham 3rd PL, Lin SX, Larson BL. A U.S. population-based survey of Staphylococcus aureus colonization. Ann Intern Med. 2006;144:318-325.

8. Baird D. Mackie and McCartney practical medical microbiology. In: Collee JG, Fraser AG, Marmion BP, Simmons A, eds. Staphylococcus: Cluster Forming Gram Positive Cocci. New York: Churchill Livingstone; 1996:245-261.

9. Clinical Laboratory Standards Institute. Performance Standards for Antimicrobial Susceptibility Testing-20th Informational Supplement. M 100-S20. Wayne (PA): CLSI; 2010.

10. Siripornmongcolchai $\mathrm{T}$, Chomvarin $\mathrm{C}$, Chaicumpar $\mathrm{K}$, Limpaiboon T, Wongkhum C. Evaluation of different primers for detecting mecA gene by PCR in comparison with phenotypic methods for discrimination of methicillinresistant Staphylococcus aureus. Southeast Asian J Trop Med Public Health. 2002;33:758-763.

11. Murakami K, Minamide W, Wada K, Nakamura E, Teraoka H, Watanabe S. Identification of methicillin-resistant strains of Staphylococci by polymerase chain reaction. J Clin Microbiol. 1991;29:2240-2244.

12. Saxena S, Singh K, Talwar V. Methicillin-resistant Staphylococcus aureus prevalence in community in the east Delhi area. Jpn J Infect Dis. 2003;56:54-56.

13. Chatterjee SS, Ray P, Aggarwal A, Das A, Sharma M. A community-based study on nasal carriage of Staphylococcus aureus. Indian J Med Res. 2009;130:742-748.

14. Sanford MD, Widmer AF, Bale MJ, Jones RN, Wenzel RP. Efficient detection and long-term persistence of the carriage of methicillin-resistant Staphylococcus aureus. Clin Infect Dis. 1994;19:1123-1128.

15. Nilsson P, Ripa T. Staphylococcus aureus throat colonization is more frequent than colonization in the anterior nares. J Clin Microbiol. 2006;44:3334-3339.

16. Krishna BV, Patil AB, Chandrasekhar MR. Community acquired methicillin resistant Staphylococcus aureus infection in a South Indian city. South Asian J Trop Med Public Health. 2004;35:371-374. 